\title{
Impact behaviour of nylon-rubber blends: 4. Effect of the coupling agent, maleic anhydride
}

\author{
R. J. M. Borggreve* and R. J. Gaymans \\ University of Twente, PO Box 217, 7500 AE Enschede, The Netherlands \\ (Received 13 April 1988; accepted 13 June 1988)
}

\begin{abstract}
Blends of $90 \mathrm{wt} \%$ nylon- 6 and $10 \mathrm{wt} \%$ EPDM (ethylene propylene diene monomer) rubber were prepared. The rubber was grafted with various amounts of maleic anhydride (MA) with the aid of a peroxide. The initial moduli and the melt viscosities of the rubbers were only a little affected by the MA and peroxide concentrations. The effect of MA concentration on the blending process and the impact behaviour was studied. The interfacial layer was investigated using infra-red spectroscopy and elemental analysis. The MA grafted onto the rubber was found to react with the nylon during the blending process. With the MA-grafted rubbers, a much finer dispersion could be obtained. However, the concentration of the coupling agent, within the range 0.13 to $0.89 \mathrm{wt} \%$ grafted onto the rubber, has hardly any influence on either the dispersion process or the impact behaviour of the blends.
\end{abstract}

(Keywords: nylon-rubber blend; maleic anhydride; blending process; impact toughness; particle size; interfacial adhesion)

\section{INTRODUCTION}

In the last decade it has been reported regularly that the impact resistance of polyamides can be improved enormously by the incorporation of small elastomeric particles into the matrix ${ }^{1-3}$. In a previous paper $^{4}$ we have shown that both the size and the concentration of the dispersed rubber phase have considerable effects on the impact strength of nylon-rubber blends. It was found that the temperature at which the transition from brittle to tough (BT) fracture behaviour occurs decreases with the interparticle distance, $I D$. The $I D$ is determined by the rubber volume fraction and the rubber particle size.

Studying the influence of the adhesion between the two phases in the blend on the impact behaviour is somewhat more complicated because changing the interfacial adhesion may have an effect on the blending process and consequently on the size of the dispersed rubber phase. $\mathrm{Wu}^{5}$ showed that a modified Taylor relationship can be applied to describe the dispersion process of rubber in nylon-6,6 in a twin-screw extruder:

$$
d=\left(\sigma_{\mathrm{i}} / \dot{\gamma} \eta_{\mathrm{m}}\right) f\left(\eta_{\mathrm{d}} / \eta_{\mathrm{m}}\right)
$$

The diameter $d$ of the dispersed phase at equilibrium is, according to this equation, directly proportional to the interfacial tension $\sigma_{i}$ and inversely proportional to the applied shear rate $\dot{\gamma}$ and the matrix viscosity $\eta_{\mathrm{m}}$. Besides, $d$ is a function of the viscosity ratio $\eta_{\mathrm{d}} / \eta_{\mathrm{m}}$, where $\eta_{\mathrm{d}}$ is the viscosity of the dispersed phase.

$\mathrm{Wu}^{5}$ demonstrated that the smallest particle size was obtained when the viscosity ratio equals unity. For his system, Wu found a linear increase of $\log d$ with $\log \left(\eta_{\mathrm{d}} / \eta_{\mathrm{m}}\right)$, provided that $\eta_{\mathrm{d}} / \eta_{\mathrm{m}} \geqslant 1$. Equation (1) denotes that decreasing the interfacial tension (by increasing the interfacial adhesion) leads to a decrease in the size of the dispersed phase, $d$. Besides, Elmendorp ${ }^{6}$ showed that coalescence of the dispersed phase during blending can only be prevented if the interfacial tension between

\footnotetext{
* Present address: DSM Research, PO Box 18, 6160 MD Geleen, The Netherlands
}

the two phases is sufficiently low. Through affecting the blending process and consequently the size of the dispersed rubber particles, the interfacial adhesion indirectly influences the impact behaviour of nylonrubber blends.

The question arises whether the adhesion between the nylon matrix and the rubber spheres also directly contributes to the impact toughness. In polymer matrices where crazing is the deformation mode (e.g. polystyrene, poly(methyl methacrylate)) strong adhesion involving interfacial chemical bonding is thought to be necessary to achieve good impact toughness because craze termination cannot operate if the rubber particles are detached from the matrix ${ }^{7,8}$.

According to $\mathrm{Wu}^{9}$ an interfacial adhesion with a tear energy caused by van der Waals attraction is enough for toughening polymers like nylon in which the deformation mode is mainly shear yielding. Jang ${ }^{10}$ found that polypropylene can be toughened by physically blending in a rubbery phase without appreciable interfacial bonding. Whether an improvement of the interfacial adhesion

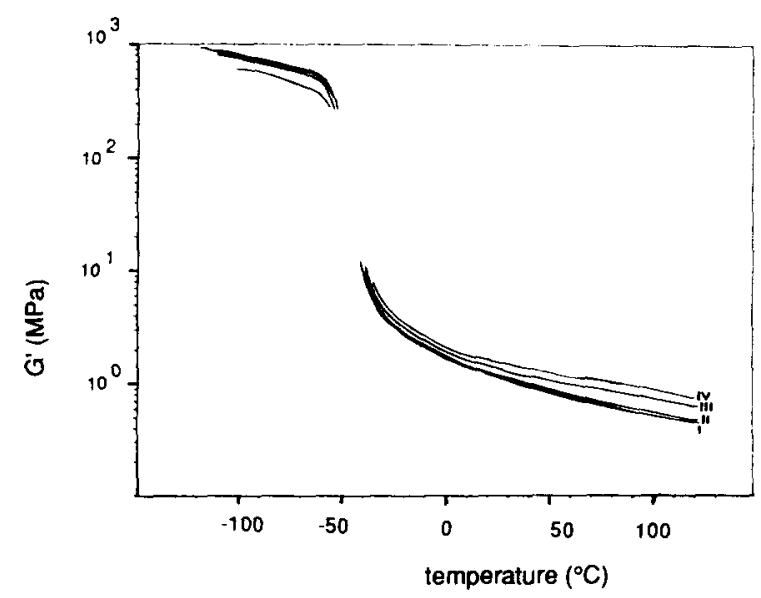

Figure $11 \mathrm{~Hz}$ shear modulus versus temperature of rubbers I to IV 


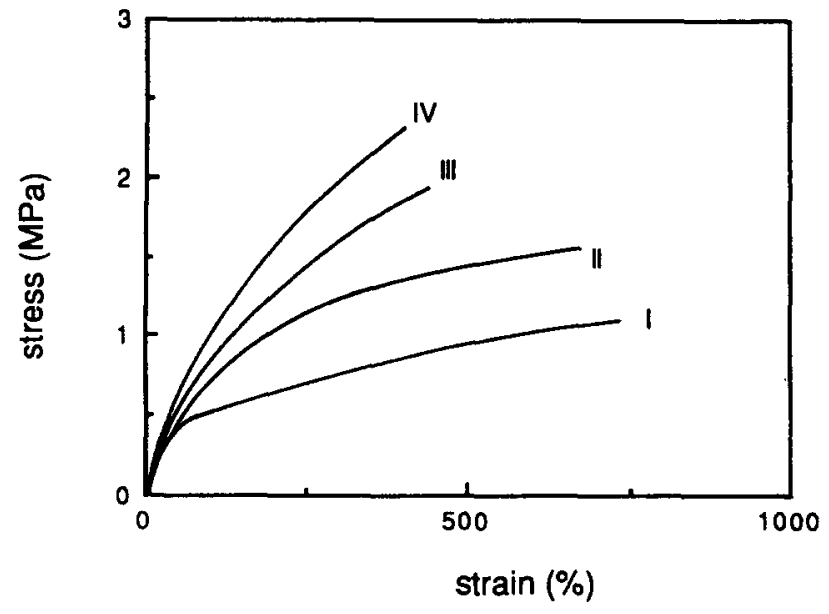

Figure 2 Stress-strain diagrams of rubbers I to IV measured at room temperature and strain rate $150 \% \mathrm{~min}^{-1}$

between rubber and a ductile matrix like polypropylene or polyamide could further improve the toughness remains unclear. However, Hobbs ${ }^{11}$ stated that the interfacial debonding energy may be an appreciable fraction of the total energy consumed during fracture of rubber-modified nylon when the average size of the dispersed particles is of the order $0.3 \mu \mathrm{m}$.

The in situ measurement of the adhesion between rubber particles and the nylon matrix is rather difficult. $\mathrm{Wu}^{9}$ related the interfacial adhesion in the blend to the peel strength of a nylon-rubber two-layer sheet, produced by compression moulding. This method is somewhat risky since it puts the mutual reactivity of two phases blended thoroughly in an extruder on one level with the chemical interaction between two compression-bounded layers.

Hert and coworkers ${ }^{12}$ tested the adhesive strength of a multilayer structure of polyamide- 6 and a terpolymer of ethylene-maleic anhydride-ethylene acrylic ester, produced by coextrusion. The peel strength of these layers was found to increase with maleic anhydride concentration. However, it was also found that the failure was cohesive, namely in the rubber phase and not at the interface. Cimmino et al. ${ }^{13}$ suggested that, during the blending of nylon- 6 and an elastomer modified with maleic anhydride, a reaction takes place between the amine end-group of the nylon and the anhydride group of the rubber, thus forming an imide bond. To prove the presence of this particular chemical bond at the blend interface is difficult because the interface layer is extremely thin. However, the graft copolymer concentration formed at the interface is essential for the interfacial adhesion and tension.

In this work an attempt is made to study the influence of the interfacial adhesion on the impact behaviour of nylon-rubber blends, independent of the particle size effect. Blends have been made of nylon- 6 with EPDM (ethylene propylene diene monomer) rubber. As a coupling agent, maleic anhydride (MA) is used. MA first is grafted onto the rubber with the aid of a peroxide in an extruder. At a certain temperature the peroxide breaks up into two radicals. A peroxide radical withdraws a hydrogen atom from the rubber molecule leaving this molecule as a radical. The rubber radical can react with a MA molecule forming a succinic anhydride group covalently bonded to the rubber. Finally, a number of termination reactions (two radicals reacting) are possible, as described by Braun $^{14}$.

In order to study the role of the interfacial adhesion in the toughness of nylon-rubber blends, the concentration of MA grafted onto the rubber is varied. The use of peroxides when grafting MA onto rubber will also have consequences for the rubber viscosity, which in its turn influences the dispersion process in the nylon. The particle size in the blends is varied by manipulating the extrusion conditions, especially the temperature and shear rate.

\section{EXPERIMENTAL}

\section{Materials}

The matrix is nylon-6, obtained from Akzo: $\mathrm{M} 258 ; \eta_{\mathrm{rel}}=6.8$ in $96 \% \mathrm{H}_{2} \mathrm{SO}_{4} ; M_{\mathrm{n}}=35.000 \mathrm{~g} \mathrm{~mol}^{-1}$. The rubber is EPDM (ethylene propylene dicyclopentadiene, 65/34/1 wt \%; Keltan 740, supplied by DSM), which has a density $\rho=0.78 \mathrm{~g} \mathrm{~cm}^{-3}$. EPDM is grafted with various amounts of maleic anhydride using Nourymix (a $50 / 50 \mathrm{wt} \%$ master batch of MA on polyethylene) and bis(t-butylperoxyisopropyl)benzene (Perkadox 14, Px14), both supplied by Akzo Chemie.

\section{Rubber modification}

The functionalization of EPDM with MA is carried out in a Brabender $19 \mathrm{~mm}$ single-screw extruder. The MA and initiator concentrations are proportionally varied. After removing of free MA by (a) washing with water (16 h at room temperature) and (b) drying overnight in a vacuum oven at $110^{\circ} \mathrm{C}$, the amount of MA grafted onto the rubber was measured with both potentiometric titration and infra-red spectroscopy.

Potentiometric titration. The reactive rubber was placed in water for $48 \mathrm{~h}$ in order to hydrolyse the anhydride groups. After drying overnight at $50^{\circ} \mathrm{C}$ the rubber was dissolved in a mixture of dichloroethane/ethanol (ratio $9 / 1$ ) at a temperature of $60^{\circ} \mathrm{C}$. By titrating with a $0.1 \mathrm{M}$ $\mathrm{KOH}$ solution in t-butanol the MA concentration could be established.

Infra-red spectroscopy. An infra-red spectrum of the reactive rubber was made using a thin compressionmoulded film. The maleic anhydride content is determined by relating the peak due to the carbonyl stretching vibration of the anhydride group at $1785 \mathrm{~cm}^{-1}$ to the $\mathrm{C}-\mathrm{H}$ stretching vibration of the $\mathrm{CH}_{2}$ group at $715 \mathrm{~cm}^{-1}$. An extensive report on this procedure is in preparation ${ }^{15}$.

\section{Rheometry}

The viscosities of nylon- 6 and the rubbers were measured at the extrusion temperatures as a function of shear rate, using a Rheometrics mechanical spectrometer: radius of the sample disc $\approx 1.25 \mathrm{~cm}$; sample thickness $\approx 0.2 \mathrm{~cm}$.

\section{Rubber testing}

The mechanical properties of the rubbers were tested with a tensile test (DIN 53455, specimen type 4, drawing speed $150 \% \mathrm{~min}^{-1}$ ) on an Instron machine. The shear moduli of the rubbers were measured with a Myrenne torsion apparatus ATM 3 at a frequency of $1 \mathrm{~Hz}$ and a heating rate of $0.5^{\circ} \mathrm{C} \mathrm{min}^{-1}$. 
Table 1 MA and Px14 concentrations added to EPDM rubber and the MA concentration grafted onto the rubber

\begin{tabular}{lllll}
\hline Rubber & $\begin{array}{l}\text { MA added } \\
(\mathrm{wt} \%)\end{array}$ & $\begin{array}{l}\text { Px14 added } \\
\text { (wt \%) }\end{array}$ & $\begin{array}{l}\text { MA grafted } \\
(\mathrm{wt} \%)\end{array}$ & $\begin{array}{l}\text { Efficiency } \\
(\%)\end{array}$ \\
\hline I & 0.5 & 0.006 & 0.13 & 26 \\
II & 1 & 0.013 & 0.25 & 25 \\
III & 2 & 0.025 & 0.43 & 22 \\
IV & 4 & 0.05 & 0.89 & 22 \\
\hline
\end{tabular}
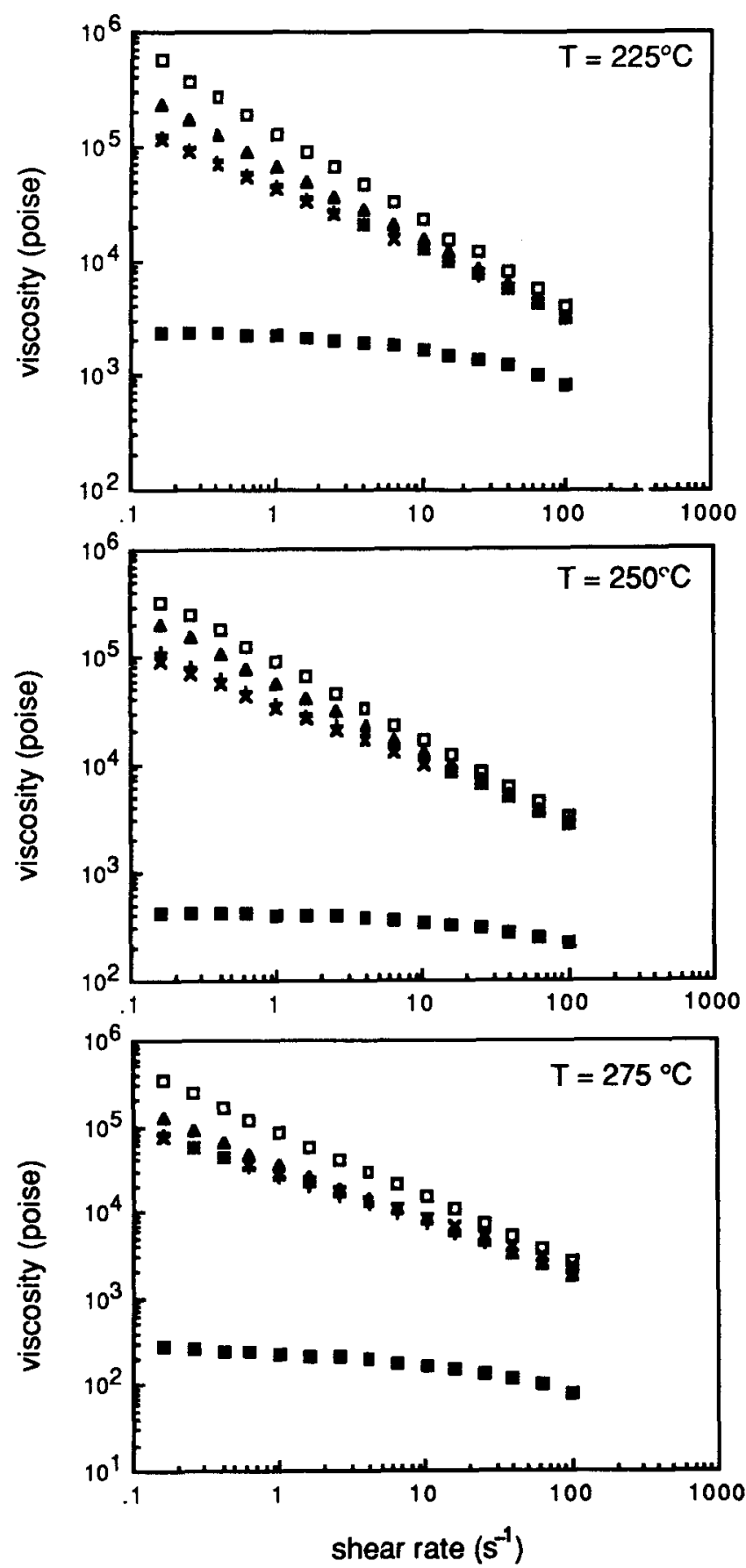

Figure 3 Viscosity versus shear rate at 225,250 and $275^{\circ} \mathrm{C}$ :

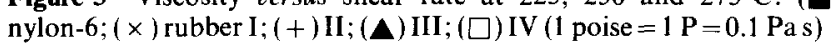

\section{Blend preparation}

A number of blends were prepared by compounding nylon-6 with the modified EPDM on a Kautex $40 \mathrm{~mm}$ single-screw extruder fitted with a $10 \mathrm{~cm}$ long cavity transfer mixing (CTM) head ${ }^{16}$, screw speed $50 \mathrm{rpm}$. Another series of blends were prepared on a Brabender $19 \mathrm{~mm}$ single-screw extruder, screw speed $20 \mathrm{rpm}$. In all the blends, the same rubber fraction was used $(10 \mathrm{wt} \%)$. Rubber particle size was changed by varying the barrel temperatures of the Brabender extruder. The blends were injection moulded on an Arburg Allrounder 221-35-250 to samples for impact testing.

\section{Scanning electron microscopy}

Smooth surfaces of the samples were prepared using a Jung microtome, equipped with a glass knife and a liquid-nitrogen cooling unit. Rubber was extracted from the surface by etching with boiling xylene overnight. The samples were furnished with a gold layer and examined with a Jeol scanning electron microscope. From the photomicrographs the particle size distribution could be obtained $^{4}$. In this study the so-called weight average particle size is used:

$$
d_{\mathrm{w}}=\sum n_{\mathrm{i}} d_{\mathrm{i}}^{2} / n_{\mathrm{i}} d_{\mathrm{i}}
$$

\section{Characterization of the interface}

In this work the interfacial adhesion is related to the amount of nylon grafted onto the rubber after the blending process. The grafting degree of the blends can be determined following the extraction procedure as proposed by Van der Velden ${ }^{17}$. The unreacted nylon is removed by dissolving in formic acid. With elemental analysis the grafting degree can then be determined by measuring the amount of nylon coupled to the rubber. The grafting degree $\alpha$ is defined as:

$$
x=\frac{\text { weight of nylon coupled to the rubber }}{\text { rubber weight }}
$$

When multiplying the grafting degree with the rubber density $\rho$ the nylon mass grafted per unit rubber volume is obtained. Dividing $\alpha \rho_{\text {rubber }}$ by the interfacial area per unit rubber volume $(=6 / d$, where $d$ is the average rubber particle diameter) gives $\beta$, the nylon mass grafted per unit interfacial area. To a first approximation $\beta$ may be considered as a measure of the interfacial adhesion since polyamides with the same molecular weight are used in this work.

In order to determine $\alpha, 5 \mathrm{~g}$ of a blend are suspended

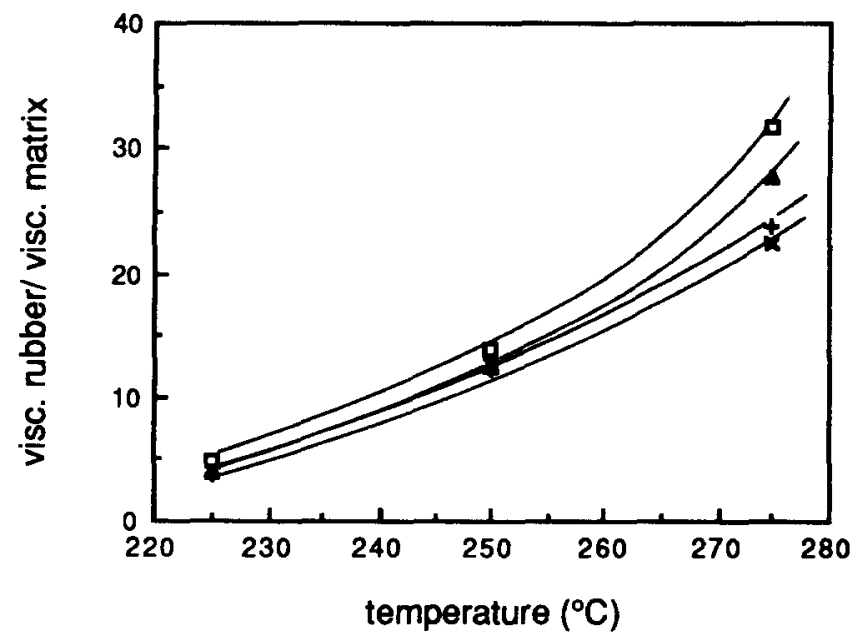

Figure 4 Viscosity ratio of rubber/nylon-6 as a function of temperature at shear rate of $100 \mathrm{~s}^{-1}$. Rubber: $(x) \mathrm{I} ;(+) \mathrm{II} ;(\boldsymbol{\Delta}) \mathrm{III}$; ( $\square$ ) IV 


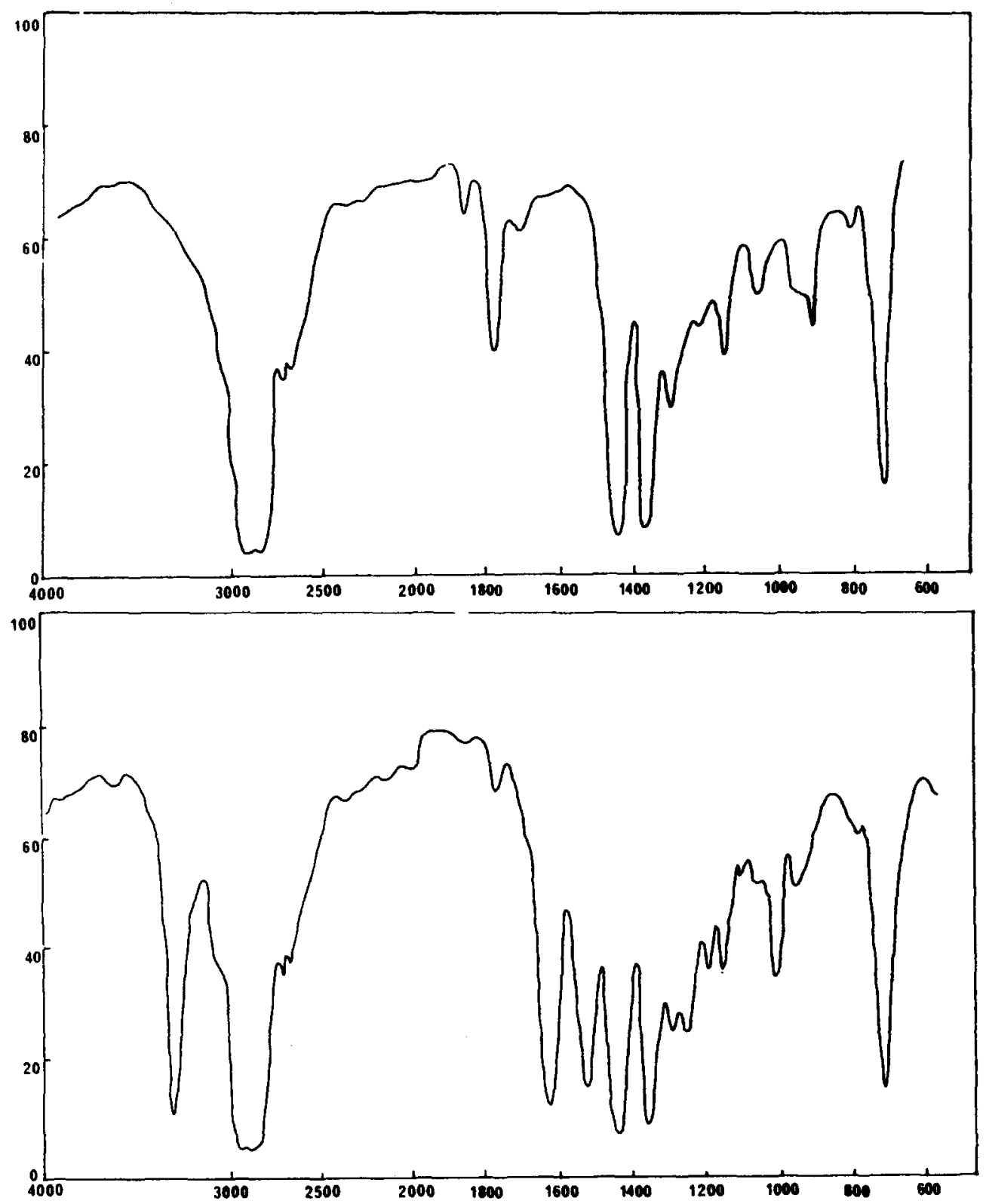

Figure 5 Infra-red spectra of (a) rubber II before blending and (b) rubber II after blending and non-coupled polyamide- 6 removed with formic acid

in $50 \mathrm{ml} 98 \%$ formic acid. The polyamide phase is in principle soluble whereas the rubber spheres are insoluble. According to Molau ${ }^{18}$ and Illing ${ }^{19}$, the formation of a white, colloidal suspension indicates that a grafting reaction has taken place. The soluble and insoluble parts of the emulsion are separated by centrifuging with a Sorvall Superspeed RC2-B using a centrifuging speed of $15000 \mathrm{rpm}$. The clear liquid at the bottom of the vessel is removed with a syringe. The liquid contains dissolved nylon, as can be proved by squirting it into water, since this is a non-solvent for polyamides.

The rubbery residue is suspended again in formic acid and the procedure is repeated three times. The last time no nylon could be isolated any more. The residual solid is washed with water and dried overnight at $90^{\circ} \mathrm{C}$ in a vacuum oven. The amount of nylon grafted onto the separated rubber is determined by elemental analysis. With a Carlo Erba Elemental Analyzer 1106, the weight per cent $\mathbf{N}$ in the rubbery residue was measured, from which the weight per cent nylon could be calculated.
With a Lauffer compression-moulding machine, thin films of the extraction products were made. An infra-red spectrum of the products is taken with a Perkin-Elmer 1310 infra-red spectrophotometer, in order to study the chemical composition of the interface material.

\section{Impact testing}

The samples were dried before testing: $24 \mathrm{~h}, 110^{\circ} \mathrm{C}$ in a vacuum oven. Notched Izod impact strength is measured (ISO 180/1A) using a Zwick impact testing machine in a temperature range from $-40^{\circ} \mathrm{C}$ up to $80^{\circ} \mathrm{C}$. The average values of at least five tests are reported.

\section{RESULTS AND DISCUSSION}

\section{Functionalization of EPDM}

The functionalization of EPDM is carried out with different amounts of MA and peroxide. Good agreement has been found between the two methods for measuring 

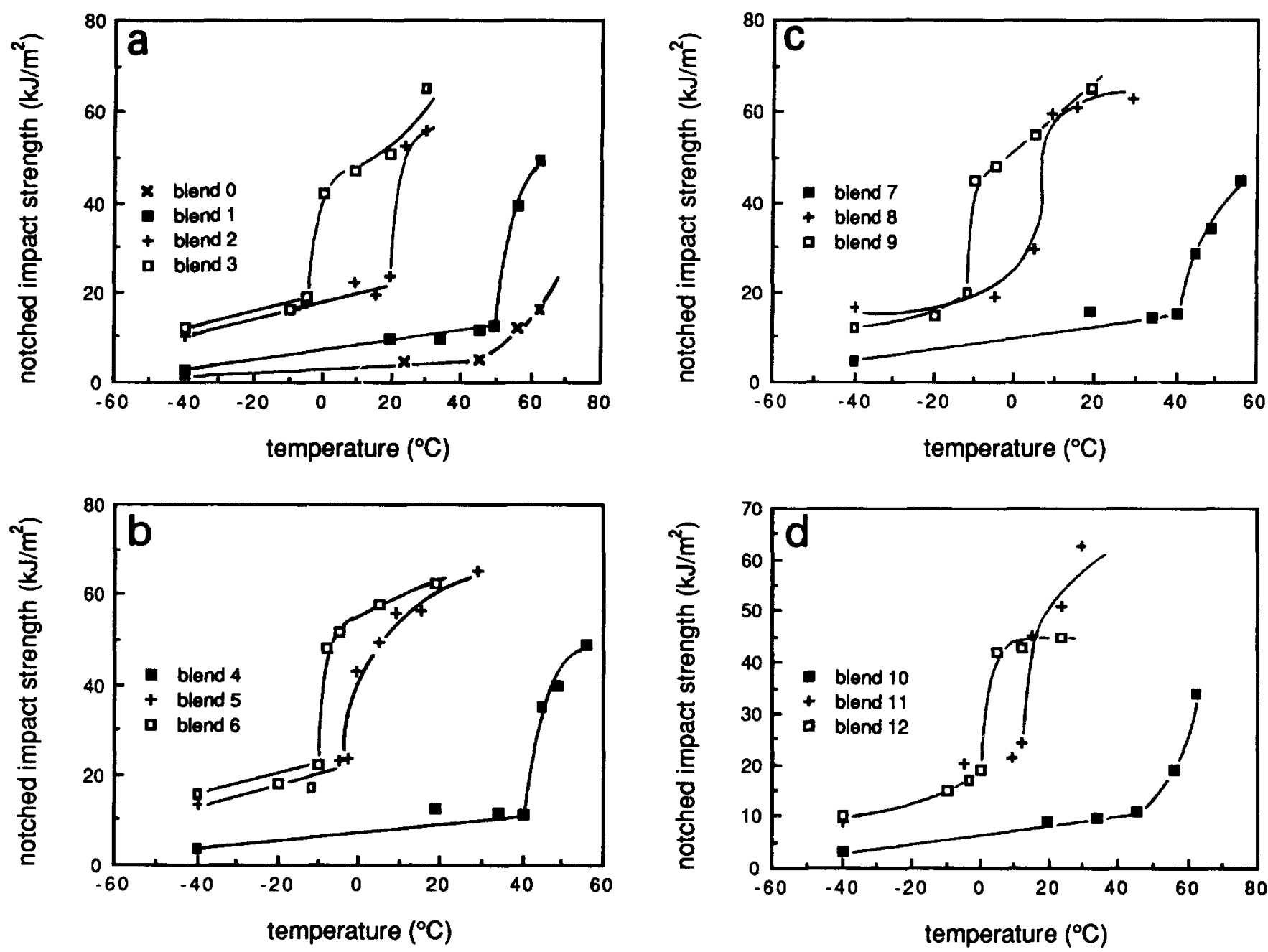

Figure 6 Notched Izod impact strengths versus temperature. (a) Blend 0, non-modified rubber; blends 1-3, rubber I. (b) Blends 4-6, rubber II. (c) Blends 7-9, rubber III. (d) Blends 10 to 12 , rubber IV

the MA content coupled to the rubber, infra-red analysis and titration. In Table 1 the results of the functionalization procedure are given. The grafting efficiency is about one-fourth for each MA and Px14 concentration added to the rubber. Without Px14 the grafting efficiency is considerably lower $(<5 \%)$. A more detailed report on the modification of EPDM rubber with maleic anhydride is in preparation ${ }^{15}$.

In Figure 1 the shear moduli of the rubbers are given as a function of temperature. The different $\mathbf{M A}$ and peroxide concentrations in the rubbers seem to have only a slight effect on $G^{\prime}$. From the data used to construct the stress-strain diagrams of Figure 2 it could be deduced that the initial modulus is hardly affected by the MA and peroxide concentrations. However, when the deformation exceeds $50 \%$, differences can be noticed; e.g. both the stress at break and the elongation at break differ with modification mode.

\section{Rheological properties of the modified rubbers and nylon-6}

The melt viscosities of nylon- 6 and the modified rubbers are plotted as functions of shear rate at 225, 250 and $275^{\circ} \mathrm{C}$ in Figure 3. As could be expected, the viscosities of the polymers and, in particular, the rubbers decrease with increasing temperature and shear rate. The viscosity of the rubbers increases with increasing peroxide concentration used for grafting: viscosity I $<$ II $<$ III $<$ IV. Especially, the viscosity of rubber IV at low shear rate is several orders higher than the other rubber viscosities. However, at high shear rate the difference diminishes. The difference in viscosity between rubbers and nylon at low shear rates is of the order of two decades at $225^{\circ} \mathrm{C}$, just above the melting temperature of nylon- 6 , moving up to approximately three decades at $275^{\circ} \mathrm{C}$.

The viscosity ratio $\eta_{\mathrm{d}} / \eta_{\mathrm{m}}$ at high shear rate $\left(100 \mathrm{~s}^{-1}\right)$ is given as a function of temperature in Figure 4. At low temperature almost no differences in viscosity ratio can be noticed between the four blend combinations. Thus, the peroxide concentration used to modify EPDM will probably not affect the process of rubber dispersion in nylon- 6 if a high shear rate and a low blending temperature are applied. Since the nylon viscosity increases and $\eta_{\mathrm{d}} / \eta_{\mathrm{m}}$ decreases with decreasing temperature, a finer dispersion of the elastomer phase can be expected at low blending temperature.

\section{Blending process}

Blends are made out of nylon- 6 and each of the four rubbers. The rubber concentration in all blends is $13 \mathrm{vol} \%$. In Table 2 the blend characteristics are given as functions of the blending procedure and the type of 
Impact behaviour of nylon-rubber blends (4): R. J. M. Borggreve et al.

Table 2 Blend characteristics as a result of the rubber, the extruder and blending temperature used

\begin{tabular}{rllllllllr}
\hline Blend & Rubber & Extruder & $\begin{array}{l}\text { Barrel } \\
\text { temp. }\end{array}$ & $\begin{array}{l}\text { Particle } \\
\text { size }(\mu \mathrm{m})\end{array}$ & $\begin{array}{l}\text { Residual } \\
\text { MA (wt \%) }\end{array}$ & $\begin{array}{l}\text { Reacted } \\
\text { MA (\%) }\end{array}$ & $\begin{array}{l}\alpha \\
(\text { wt \%) }\end{array}$ & $\begin{array}{l}\beta \\
\left(\mathrm{mg} \mathrm{m}^{-2}\right)\end{array}$ & $\begin{array}{l}M_{\mathrm{n}}^{*} \\
\left(\mathrm{~g} \mathrm{~mol}^{-1}\right)\end{array}$ \\
\hline 0 & EPDM & K & L & $>5$ & - & - & 0 & 0 & - \\
1 & I & B & H & 1.98 & 0.02 & 85 & 2 & 5.1 & 2000 \\
2 & I & B & L & 0.71 & 0.02 & 77 & 7 & 6.6 & 7000 \\
3 & I & K & L & 0.31 & 0.03 & 77 & 16 & 6.4 & 16000 \\
4 & II & B & H & 1.53 & 0.09 & 64 & 3 & 5.9 & 2000 \\
5 & II & B & L & 0.37 & 0.10 & 80 & 15 & 7.2 & 8000 \\
6 & II & K & L & 0.25 & 0.08 & 68 & 18 & 5.9 & 11000 \\
7 & III & B & H & 0.94 & 0.11 & 74 & 7 & 8.6 & 2000 \\
8 & III & B & L & 0.46 & 0.09 & 77 & 15 & 8.9 & 5000 \\
9 & III & K & L & 0.29 & 0.03 & 93 & 29 & 10.9 & 7000 \\
10 & IV & B & H & 1.59 & 0.26 & 70 & 6 & 12.4 & 1000 \\
11 & IV & B & L & 0.66 & 0.37 & 56 & 18 & 15.4 & 4000 \\
12 & IV & K & L & 0.51 & 0.21 & 76 & 19 & 12.6 & 3000 \\
\hline
\end{tabular}

$\mathrm{K}=$ Kautex; $\mathbf{B}=$ Brabender

$\mathrm{L}=$ barrel temperatures $220-220-220-220^{\circ} \mathrm{C}$

$\mathrm{H}=$ barrel temperatures $220-250-275-275^{\circ} \mathrm{C}$

Weight-average particle size in micrometres

Residual MA $=$ MA $(w t \%)$ left in rubber after blending

Reacted MA $=\left\{\left([\mathrm{MA}]_{\text {rubber }}-[\mathrm{MA}]_{\text {residual }}\right) /[\mathrm{MA}]_{\text {rubber }}\right\} \times 100 \%$

$\alpha=$ grafting degree

$\beta=$ polyamide mass coupled at the rubber interface

$M_{\mathrm{n}}^{*}=$ molecular weight of grafted polyamide

rubber used. Blend 0, consisting of nylon- 6 and 13 vol\% non-modified EPDM Keltan 740, has been produced as a reference.

When no coupling agent is used, the resulting rubber particle size is large. The particle size is drastically decreased when the rubber has been modified with the coupling agent, maleic anhydride. In that case a grafting reaction at the nylon-rubber interface probably causes a significant decrease of the interfacial tension, which in turn implies a smaller size of the dispersed phase (equation (1)).

However, the dispersion process is, within the range studied, only slightly affected by the MA concentration grafted onto the rubber. The average particle size of the blends using rubber IV, the one with the highest MA content, is even slightly larger than those of the other blends when applying the same processing conditions. This might be due to the corresponding higher rubber viscosity. For the four modified rubbers used, the smallest particle size each time is obtained with the Kautex extruder equipped with the cavity transfer mixing head. However, a relatively fine dispersed rubber phase has also been obtained with the Brabender, using the low-temperature barrel profile so that the matrix viscosity has been kept relatively high and the viscosity ratio $\eta_{\mathrm{d}} / \eta_{\mathrm{m}}$ low (Figure 4). The MA content left in the modified rubber after blending can be roughly measured after the non-reacted polyamide has been extracted from the blend with formic acid. The chemical composition of the rubbery residue can then be studied by infra-red spectroscopy. A typical i.r. spectrum of such a residue is shown in Figure 5. Compared to the modified rubber before blending, the presence of a polyamide is clear and, moreover, it can be seen that the MA peak at $1785 \mathrm{~cm}^{-1}$ has been strongly reduced, which implies that the MA content in the rubber has considerably diminished. The disappearance of MA suggests that a reaction during the blending process took place. Unfortunately, since the grafted polyamide strongly dominates the spectrum there is no decisive answer to what kind of chemical bond has been formed at the interface. However, the absence of a strong free-acid bond at $1720 \mathrm{~cm}^{-1}$ suggests that a graft copolymer with an imide bond has been formed rather than an amide/acid graft copolymer. This would support the conclusions of Cimmino's model study ${ }^{13}$. The residual MA concentrations are given in Table 2, as well as the per cent MA which has reacted. Remarkably, in all blends approximately the same per cent maleic anhydride $(60-80 \%)$ has disappeared. This figure seems to be independent of initial MA concentration, blend conditions or resulting particle size. Whereas the determination of the MA concentration in the rubbery residue with infra-red measurements is rather crude, the polyamide concentration can be measured accurately with elemental analysis. If no coupling agent is used, no polyamide is found to be grafted. When MA modified rubbers are used, the weight per cent polyamide grafted onto the rubber, $\alpha$, increases with decreasing particle size or, stated otherwise, increasing interfacial area.

From $\alpha$ and the mass of MA which has reacted, a rough estimation can be made of the molecular weight of the grafted polyamide. The lower $M_{\mathrm{n}}$ of the grafted polyamide (Table 2) in comparison with the $M_{\mathrm{n}}$ of the bulk ( $35000 \mathrm{~g} \mathrm{~mol}^{-1}$ ) suggests that the MA grafted onto the rubber has reacted with amide groups of the nylon molecules, consequently inducing chain scission. It is notable that the $M_{\mathrm{n}}$ of the grafted polyamide is lowest when the high extrusion temperature was applied.

It appears that $\beta$, the amount of polyamide coupled to the rubber per unit interfacial area, increases with increasing MA concentration on the rubber. However, almost no difference in $\beta$ can be noticed between the rubbers with the lowest MA concentrations, I and II.

Thus, the rubber dispersion process in nylon-6 drastically improves when introducing MA as a coupling agent. Increasing the amount of MA grafted onto the 

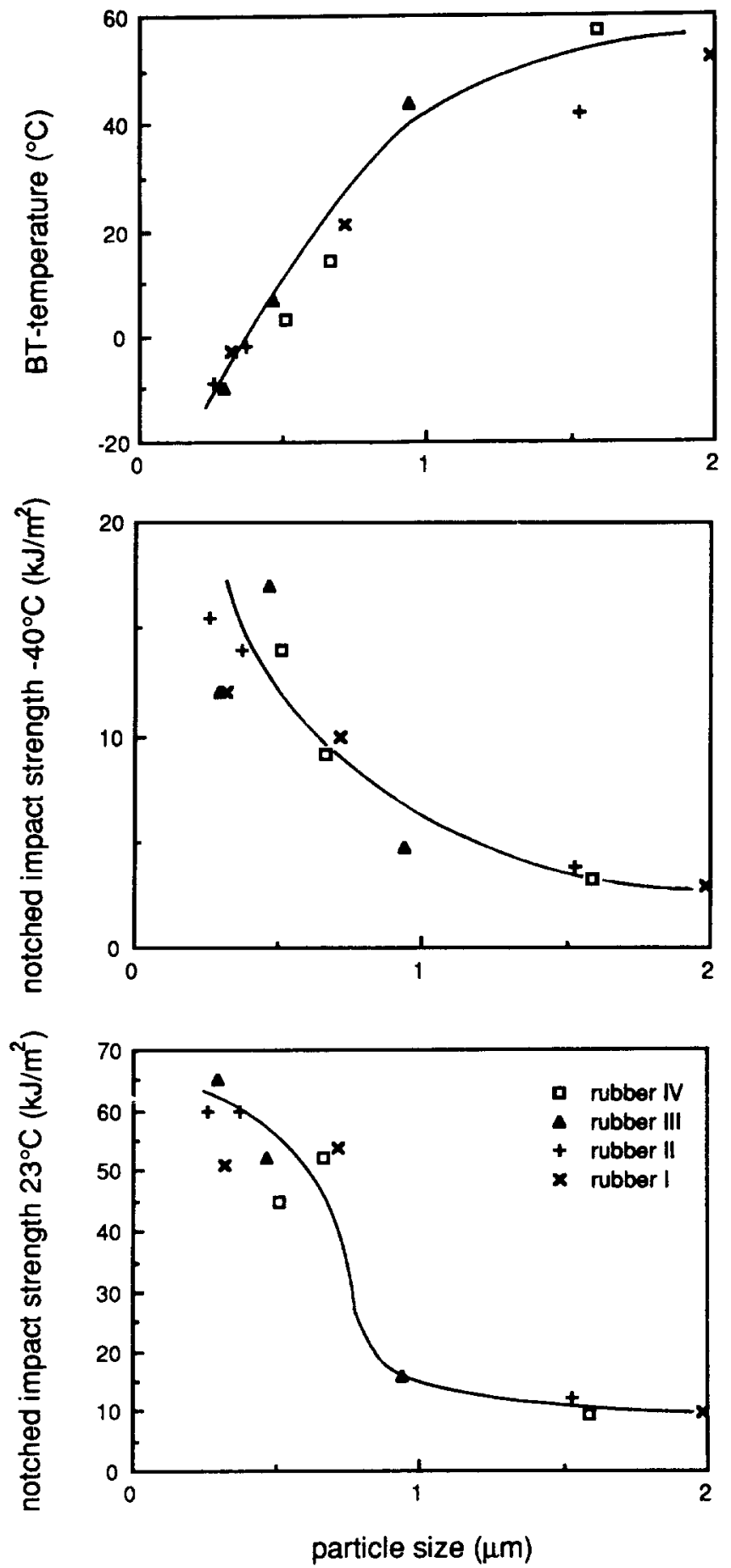

Figure 7 BT temperature and notched Izod impact strengths at -40 and $23^{\circ} \mathrm{C}$ as functions of the weight-average rubber particle size for blends 1 to 12 . The different symbols refer to the type of rubber in the blend

rubber seems to increase moderately the amount of polyamide grafted at the polyamide-elastomer interface after blending but, within the range studied, it seems that the MA concentration has no significant effect on the size of the dispersed phase.

\section{Impact properties}

The notced Izod impact strength as a function of temperature of each blend is plotted in Figure 6. For each blend, at a certain temperature a typical brittle-tough (BT) transition ${ }^{4}$ is found. In order to discriminate between the effects of particle size and interfacial adhesion on the impact behaviour, the BT temperature and the Izod impact strengths at room temperature and at $-40^{\circ} \mathrm{C}$ are given respectively as functions of the weight-average particle size in Figure 7 and as functions of $\beta$ in Figure 8 . Figure 7 shows that the BT temperature decreases with decreasing particle size while the Izod impact strength at $-40^{\circ} \mathrm{C}$ increases. This is in agreement with earlier findings ${ }^{4}$. When the particle size exceeds $1.0 \mu \mathrm{m}$ the BT temperature reaches the glass transition temperature of dry nylon-6. Blends with a particle size smaller than
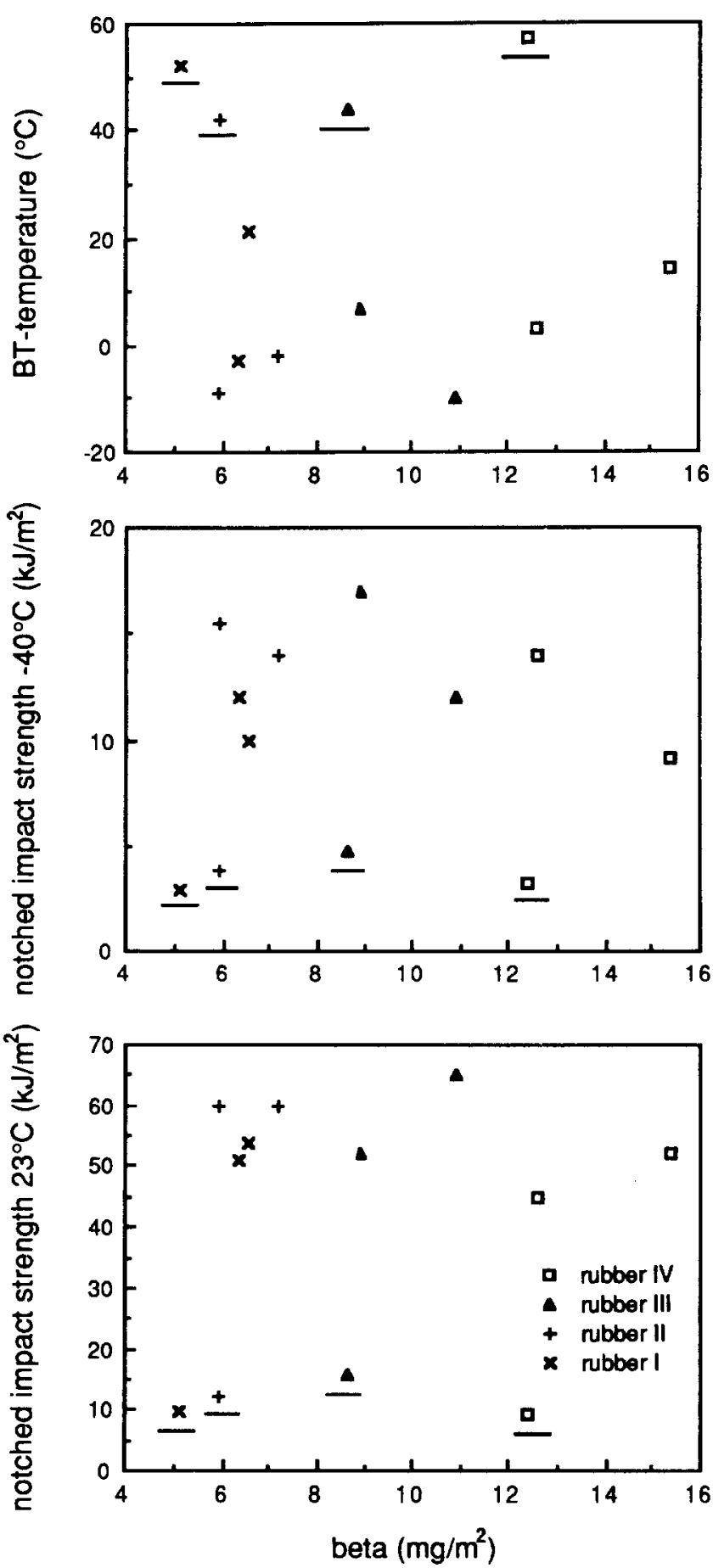

Figure 8 BT temperature and notched Izod impact strengths at -40 and $23^{\circ} \mathrm{C}$ as functions of $\beta$, the polyamide mass coupled to the elastomer per unit interfacial area for blends 1 to 12. The different symbols refer to the type of rubber in the blend. Underline symbols: weight-average rubber particle size in blend $>1 \mu \mathrm{m}$ 
$0.7 \mu \mathrm{m}$ are tough at room temperature. The relationships in Figure 7 seem to be independent of the amount of MA used in the blending process.

Moreover, Figure 8 demonstrates that the amount of polyamide grafted onto the rubber particles has no effect on the impact behaviour of nylon-rubber blends since both the BT temperature and the Izod impact strengths at $23^{\circ} \mathrm{C}$ and $-40^{\circ} \mathrm{C}$ remain on the same level with various $\beta$ and an approximately constant particle size. It should be noted that the molecular weight of the grafted polyamide, which varies considerably among the blends studied (Table 2), may have an effect on the interfacial adhesion too. Nevertheless, since the variation in molecular weight in relation to $\beta$ is rather random, it will probably not affect the essence of the conclusions.

The considerable differences in stress and elongation at break of the four elastomers (Figure 2) do not seem to affect the impact behaviour of the blends. In forthcoming work ${ }^{20,21}$ the role of the mechanical properties of the rubber in the toughening mechanism of nylonrubber blends is discussed.

\section{CONCLUSIONS}

On modifying EPDM rubber with maleic anhydride, it turns out that: (i) EPDM can easily be modified with MA in an extruder; (ii) the grafting efficiency is, at constant MA/peroxide concentration ratio, independent of MA and peroxide concentration; and (iii) at a high shear rate $\left(100 \mathrm{~s}^{-1}\right)$ and a low blending temperature, the rubber viscosity is not influenced by the peroxide concentration.

The use of MA as a coupling agent strongly improves the dispersion of EPDM rubber in nylon-6. The maleic anhydride concentration grafted onto EPDM rubber, however, within the range 0.13 to $0.89 \mathrm{wt} \%$, appears to have hardly any effect on the dispersion process in highly viscous nylon-6. Nevertheless, increasing the MA content increases the amount of polyamide grafted onto the rubber per unit interfacial area.

There is some evidence that, during blending, the MA on the rubber mainly reacts with the amide groups of the nylon, inducing chain scission. The results of the impact tests show that, within the range studied, the MA concentration used as well as the amount of polyamide coupled at the interface have no influence on the impact behaviour of nylon-rubber blends.

\section{ACKNOWLEDGEMENTS}

This work is part of the research programme of the University of Twente and was financially supported by the Netherlands Technology Foundation (STW) and by the DSM Research Company.

\section{REFERENCES}

Flexman, E. A. Polym. Eng. Sci. 1979, 19(8), 564

Brassat, W. and Nielinger, W. Kunststoffe 1982, 71, 172

Schaaf, S. Kunstst. Plast. (Solothurn, Switz.) 1983, 30, 7

4 Borggreve, R. J. M., Gaymans, R. J., Schuijer, J. and Ingen Housz, J. F. Polymer 1987, 28, 1489

5 Wu, S. Polym. Eng. Sci. 1987, 27(5), 335

6 Elmendorp, J. J. PhD Thesis, University of Technology, Delft, 1986

7 Bucknall, C. N. in 'Polymer Blends' (Eds. D. R. Paul and S. Newman), Academic Press, New York, 1978, Vol. 1

8 Kambour, R. P. J. Polym. Scil-Macromol. Rev. 1973, 7, 1

9 Wu, S. Polymer 1985, 26, 1855

10 Jang, B. Z. Polym. Eng. Sci. 1985, 25(10), 643

11 Hobbs, S. Y., Bopp, R. C. and Watkins, V. H. Polym. Eng. Sci. 1983, 23, 1983

12 Hert, M., Guerdoux, L. and Lebez, J. Angew. Makromol. Chem. 1987, 154, 111

13 Cimmino, S., D’Orazio, I., Greco, R., Maglio, G., Malinconico, M., Mancarella, M., Martuscelli, E., Palumbo, R. and Ragosta, G. Polym. Eng. Sci. 1984, 24, 48

14 Braun, D. and Eisenlohr, U. Angew. Makromol. Chem. 1976, 55, 43

15 Gaymans, R. J., Oostenbrink, B. and Borggreve, R. J. M. in preparation

16 Gale, G. M. Plast. Rubber Process. Appl. 1982, 2, 347

17 Van der Velden, G. Macromolecules, submitted

18 Molau, G. E. Kolloid Z. Z. Polym. 1970, 238, 493

19 Illing, G. Angew. Makromol. Chem. 1981, 95, 83

20 Borggreve, R. J. M., Gaymans, R. J. and Schuijer, J. Polymer $1989, \mathbf{3 0}, 71$

21 Borggreve, R. J. M., Gaymans, R. J. and Eichenwald, H. M. Polymer $1989,30,78$ 